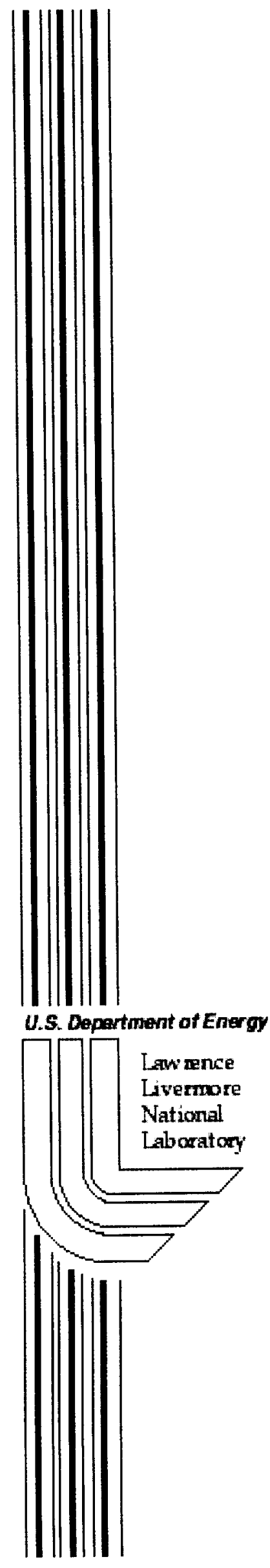

UCRL-ID-153671

\title{
Investigation of the Electronic Structure of Solid Density Plasmas by $X$-ray Scattering
}

G. Gregori, S. H. Glenzer, F. J. Forest, S. Kuhlbrodt, $R$. Redmer, G. Faussurier, C. Blancard, P. Renaudin, O. L. Landen

May 19, 2003 
This document was prepared as an account of work sponsored by an agency of the United States Government. Neither the United States Government nor the University of California nor any of their employees, makes any warranty, express or implied, or assumes any legal liability or responsibility for the accuracy, completeness, or usefulness of any information, apparatus, product, or process disclosed, or represents that its use would not infringe privately owned rights. Reference herein to any specific commercial product, process, or service by trade name, trademark, manufacturer, or otherwise, does not necessarily constitute or imply its endorsement, recommendation, or favoring by the United States Government or the University of California. The views and opinions of authors expressed herein do not necessarily state or reflect those of the United States Government or the University of California, and shall not be used for advertising or product endorsement purposes. 


\title{
Investigation of the electronic structure of solid density plasmas by x-ray scattering
}

\author{
G. Gregori, ${ }^{*}$ S. H. Glenzer, ${ }^{*}$ F. J. Forest, ${ }^{*}$ S. Kuhlbrodt, ${ }^{\dagger}$ R. Redmer ${ }^{\dagger}$ \\ G. Faussurier ${ }^{\ddagger}$ C. Blancard ${ }^{\ddagger}$ P. Renaudin,$\ddagger$ and O. L. Landen* \\ (Dated: May 19, 2003)
}

\begin{abstract}
We present an improved analytical expression for the $x$-ray dynamic structure factor from a dense plasma which includes the effects of weakly bound electrons. This result can be applied to describe scattering from low to moderate $\mathrm{Z}$ plasmas, and it covers the entire range of plasma conditions that can be found in inertial confinement fusion experiments, from ideal to degenerate up to moderately coupled systems. We use our theory to interpret $x$-ray scattering experiments from solid density carbon plasma and to extract accurate measurements of electron temperature, electron density and charge state. We use our experimental results to validate various equation-of-state models for carbon plasmas.
\end{abstract}

\section{INTRODUCTION}

$\mathrm{X}$-ray scattering of solid density plasmas has been recently proven a successful technique for the characterization of low-Z warm and dense states of matter [13]. In particular, it was shown that by extending the theory of spectrally resolved Thomson scattering in the hard x-ray regime, accurate measurements of the electron temperature, electron density and ionization state can be obtained. In this respect, comparison of the experimental results with equation of state (EOS) models has started revealing important insights on the microscopic electronic state of solid density beryllium plasmas [2]. In this paper, we present a generalization of the technique to higher $\mathrm{Z}$ materials, thus allowing the study of basic plasma parameters and transport properties of a vast range of plasma regimes, as the ones created in inertial confinement fusion (ICF) experiments [4] and found in the interior of stars and planets.

In the case of low- $\mathrm{Z}$ materials, the $\mathrm{x}$-ray dynamic form factor, which is the fundamental quantity describing the scattering cross section, is considerably simplified since it contains only two major contributions that arise from scattering from free electrons and tightly bound electrons. The first term is usually described within the random phase approximation (RPA) $[5,6]$ and it refers to photon scattering from density fluctuations of the free electrons in the plasma. During the process, energy is exchanged from the photons to the electrons, and the scattered photons are downshifted in energy by the Compton effect. Coherent scattering from tightly bound electrons, instead, is well separated in energy from the free electron term and it arises from photons that elastically scatter electrons, as energy transfer is not kinematically allowed in their bound states. The $\mathrm{x}$-ray scattering cross section

\footnotetext{
${ }^{*}$ Lawrence Livermore National Laboratory, University of California, P.O. Box 808, CA 94551

†Universität Rostock, Fachbereich Physik, Universitätsplatz, 3, D18051, Rostock, Germany

‡Département de Physique Théorique et Appliquée, CEA/DAM Ile-de-France, BP12, 91680 Bruyéres-le-Châtel Cedex, France
}

can be thus obtained for solid density matter, accounting for both ideal and quantum degenerate plasmas [1]. The transition from ideal to degenerate states often encompasses weakly or strongly coupled states [7] which may exhibit a modified response in the electron density fluctuation dynamics. A discussion on such conditions and their effect on the $x$-ray scattering form factor has been presented by Gregori et al. [8].

$\mathrm{X}$-ray scattering from moderate to high- $\mathrm{Z}$ materials add another term to the total form factor: scattering from weakly bound electrons. Since, for those electrons Compton scattering is kinematically permitted, the incident $\mathrm{x}$-ray photons have a finite probability to transfer a portion of their energy to the electrons, resulting in the appearance of a secondary feature in the spectrum of the scattered radiation that overlaps to the free electron one. Thus, the interplay of the scattering from all of these terms: free, tightly bound and weakly bound electrons, provides a unique method for a full characterization of the electronic state of the dense plasma. The number of valence (or delocalized) electrons can be directly inferred from the experimental spectra, as well as electron temperature and density, providing important EOS model validation. We will further discuss this point using carbon as an example. By extracting carbon EOS data from experimental $\mathrm{x}$-ray scattering spectra from solid density carbon plasmas, we will directly compare our results with various ionization balance models.

\section{THEORY}

Following the discussion in Gregori et al. [1], we describe the scattering from a uniform plasma containing $N$ ions per unit volume. If $Z_{A}$ is the nuclear charge of the ion, the total number of electrons per unit volume in the system, including free and bound ones, is $Z_{A} N$. Let us now assume we probe such a system with $x$-rays of frequency $\omega_{0}$ such that $\hbar \omega_{0} \gg E_{I}$, with $E_{I}$ the ionization energy of any bound electron, i.e., the incident frequency must be large compared to any natural absorption frequency of the scattering atom, which allows us to neglect photoabsorption. During the scattering process, 
the incident photon transfers, on average, momentum $\hbar \mathbf{k}$ and energy $\hbar \omega=\hbar^{2} k^{2} / 2 m_{e}=\hbar \omega_{0}-\hbar \omega_{1}$ to the electron, where $\omega_{1}$ is the frequency of the scattered radiation, and in the non-relativistic limit $\left(\hbar \omega \ll \hbar \omega_{0}\right)$

$$
k=|\mathbf{k}|=\frac{4 \pi}{\lambda_{0}} \sin (\theta / 2)
$$

with $\lambda_{0}$ the probe wavelength and $\theta$ the scattering angle. We denote with $Z_{f}$ and $Z_{c}$ the number of kinematically free and core electrons, respectively. Clearly, $Z_{A}=Z_{f}+Z_{c}$. Here $Z_{c}$ includes both tightly bound and weakly bound electrons, as there is not a net distinction between them, and for any given bound electron in the outermost shells there is a finite probability of either elastic or inelastic scattering. Since $Z_{f}$ represents electrons which are not bound to any single atom, we will also refer to it as the number of delocalized, or valence, electrons. Following the approach of Chihara $[9,10]$ the scattering cross section is described in terms of the dynamic structure factor of all the electrons in the plasma

$$
\frac{d^{2} \sigma}{d \Omega d \omega}=\sigma_{T} \frac{k_{1}}{k_{0}} S(k, \omega),
$$

where $\sigma_{T}$ is the usual Thomson cross section and $S(k, \omega)$ is the total dynamic structure factor given by

$$
\begin{array}{r}
S(k, \omega)=\left|f_{I}(k)+q(k)\right|^{2} S_{i i}(k, \omega)+Z_{f} S_{e e}^{0}(k, \omega) \\
+Z_{c} \int \tilde{S}_{c e}\left(k, \omega-\omega^{\prime}\right) S_{s}\left(k, \omega^{\prime}\right) d \omega^{\prime} .
\end{array}
$$

As extensively discussed in Gregori et al. [1], the first term in Eq. (3) accounts for the density correlations of electrons that dynamically follow the ion motion. This includes both the core electrons, represented by the ion form factor $f_{I}(k)$, and the screening cloud of free (and valence) electrons that surround the ion, represented by $q(k)[11] . S_{i i}(k, \omega)$ is the ion-ion density correlation function. The second term in Eq. (3) gives the contribution in the scattering from the free electrons that do not follow the ion motion. Here, $S_{e e}^{0}(k, \omega)$ is the high frequency part of the electron-electron correlation function [12] and it reduces to the usual electron feature $[13,14]$ in the case of an optical probe. Inelastic scattering by core electrons is included in the last term of Eq. (3), which arises from bound-free transitions to the continuum of core electrons within an ion, $\tilde{S}_{\mathrm{ce}}(k, \omega)$, modulated by the self-motion of the ions, represented by $S_{s}(k, \omega)$.

In Ref. [1], we have presented simplified expressions for each term in Eq. (3) for low- $\mathrm{Z}$ materials. In those cases, the bound-free contribution is small under most experimental conditions and it can be neglected. However, in the case of carbon, L-shell inelastic scattering needs to be included. Differently from the approach followed in Ref. [1], we propose a more comprehensive treatment of the core electron term based on the impulse approximation (IA) $[15,16]$. The IA assumes that the electronphoton interaction occurs on a very short time-scale, so the target electron always sees the same nuclear potential just before and after the collision. Since only changes in the kinetic energy needs to be considered, the electron can be treatled as free and its final energy depends on the projection of the electron's initial momentum on the scattering vector $\mathbf{k}$. Thus, the doppler broadening of the scattered radiation is proportional to the initial momentum distribution of the electron [17]. In the hydrogenic approximation for the initial wavefunction and momentum distribution of the electron, the IA profiles for $K$ and L-shells assume the form [18]

$$
\begin{gathered}
J_{1,0}^{0}(\xi)=\frac{8}{3 \pi Z_{*}\left(1+\xi^{2} / Z_{*}^{2}\right)^{3}}, \\
J_{2,0}^{0}(\xi)=\frac{64}{\pi Z_{*}}\left[\frac{1}{3\left(1+4 \xi^{2} / Z_{*}^{2}\right)^{3}}\right. \\
\left.-\frac{1}{\left(1+4 \xi^{2} / Z_{*}^{2}\right)^{4}}+\frac{4}{5\left(1+4 \xi^{2} / Z_{*}^{2}\right)^{5}}\right] \\
J_{2,1}^{0}(\xi)=\frac{64}{15 \pi Z_{*}} \frac{1+20 \xi^{2} / Z_{*}^{2}}{\left(1+4 \xi^{2} / Z_{*}^{2}\right)^{5}}
\end{gathered}
$$

where,

$$
\xi=\frac{m_{e} a_{B}}{\hbar k}\left(\omega-\frac{\hbar k^{2}}{2 m_{e}}\right)
$$

with $a_{B}$ the Bohr radius and $Z_{*}=Z_{A}-z_{n, l}$ the effective nuclear charge seen by the electron in the quantum state $n, l$. The screening constants $z_{n, l}$ depend on the atomic (or ionic) state of the atom and they can be calculated from the prescription of Pauling and Sherman [19].

As discussed by Eisenberger and Platzman [15], the IA is correct to the order of $\left(E_{B} / E_{c}\right)^{2}$, where $E_{B}$ is the binding energy and $E_{c}$ is the Compton recoil. For our typical experimental conditions, $E_{c} \sim 70 \mathrm{eV}$ and the binding energy of L-shell carbon electrons is $E_{B} \sim 11-64 \mathrm{eV}$ (depending on the ionization state), thus errors introduced by the IA can be significant. Even if $\mathrm{K}$-shell contribution is typically less important than the L-shell one, corrections to the IA need to be accounted for K-shell electrons as well. The main modification in the IA appears as a shift of the peak of feature from the free electron value, an effect known as the Compton defect (see e.g., [20, 21]). Since the IA assumes plane waves as the final state for the electron, improvement in the model can be obtained by using the first Born approximation and hydrogenic wavefunctions for both initial and final states [15, 22, 23], or by a perturbation expansion of the final states $[24,25]$. In our work we will follow the perturbative approach of Holm and Ribberfors [25] which gives for the first order asymmetric correction to the IA:

$$
J_{1,0}^{1}(\xi)=J_{1,0}^{0}(\xi) \frac{Z_{*}}{k a_{B}}\left[\frac{3}{2} \xi-2 \operatorname{atan}(\xi)\right]
$$




$$
\begin{gathered}
J_{2,0}^{1}(\xi)=J_{2,0}^{0}(\xi) \frac{Z_{*}}{k a_{B}}\left[\frac{5}{4} \frac{1+48 \xi^{4}}{1-10 \xi^{2}+40 \xi^{4}} \xi-2 \operatorname{atan}(2 \xi)\right] \\
J_{2,1}^{1}(\xi)=J_{2,1}^{0}(\xi) \frac{Z_{*}}{k a_{B}}\left[\frac{2}{3} \frac{10+60 \xi^{2}}{1+20 \xi^{2}} \xi-2 \operatorname{atan}(2 \xi)\right]
\end{gathered}
$$

with the corrected IA profiles given by $J_{n, l}(\xi)=J_{n, l}^{0}(\xi)+$ $J_{n, l}^{1}(\xi)$. Even if such modified IA expressions already provide accurate profiles for the experimental conditions of interest, further improvements to the IA can be obtained if more realistic wavefunctions (such as Hartree-Fock) are used instead of hydrogenic ones (see e.g., [20]). For Lshell electrons and atoms in their neutral state, the error introduced by the hydrogenic wavefunctions is $\$ 10 \%$ when compared to Hartree-Fock wavefunctions [26] for our typical experimental conditions.

The total bound-free dynamic structure is thus written as

$$
\tilde{S}_{c e}(k, \omega)=\frac{r_{k}}{Z_{c} B^{3}} \sum_{n, l} J_{n, l}(\xi)
$$

with the sum running over all the bound electrons. The normalization constant $r_{k}$ accounts for the possibility of coherent scattering [27] and it is given by [28]

$$
r_{k}=1-\frac{1}{Z_{c}} \sum_{n} f_{n}^{2}(k) \sim 1-\frac{f_{I}(k)}{Z_{c}^{2}}
$$

where $f_{n}(k)$ are the partial form factors for each bound electron [19] and $\sum_{n} f_{n}(k)=f_{I}(k)$. The coefficient $B$ in eq. (11) is only important for very large momentum transfer and it is given by the Breit-Dirac formula [28]

$$
B=1+\frac{1}{\omega_{0}} \frac{\hbar k^{2}}{2 m_{e}}
$$

Profiles of the bound-free dynamic structure for a carbon plasma are given in Figure 1 for different ionization states and typical experimental conditions. The carbon is assumed to be in an amorphous state (foam) with density $0.72 \mathrm{~g} / \mathrm{cc}$. In the high frequency limit, the ion-ion self structure is $S_{s}(k, \omega) \sim \delta(\omega)$, as ion dynamics remain unresolved under our experimental conditions [1]. Since bound-free transitions are not allowed if $\hbar \omega<E_{B}$, the dynamic factor (11) has a cut-off at the ionization energy for L-shell electrons, as it is clearly shown in Figure 1. Similarly, for K-shell electrons, the cut-off marks the $\mathrm{K}$-shell binding energy.

In the case of very dense plasma, the potential distribution of a given ion is influenced not only by its own bound electrons but also by the neighboring ions. The net effect is a lowering of the ionization potential (continuum lowering). Such lowering depends on the total number of ions that participate in the modification of the potential around a test ion, which, in turn, is a function of the screening distance of the Coulomb forces. Stewart and Pyatt [29] have calculated the continuum lowering using

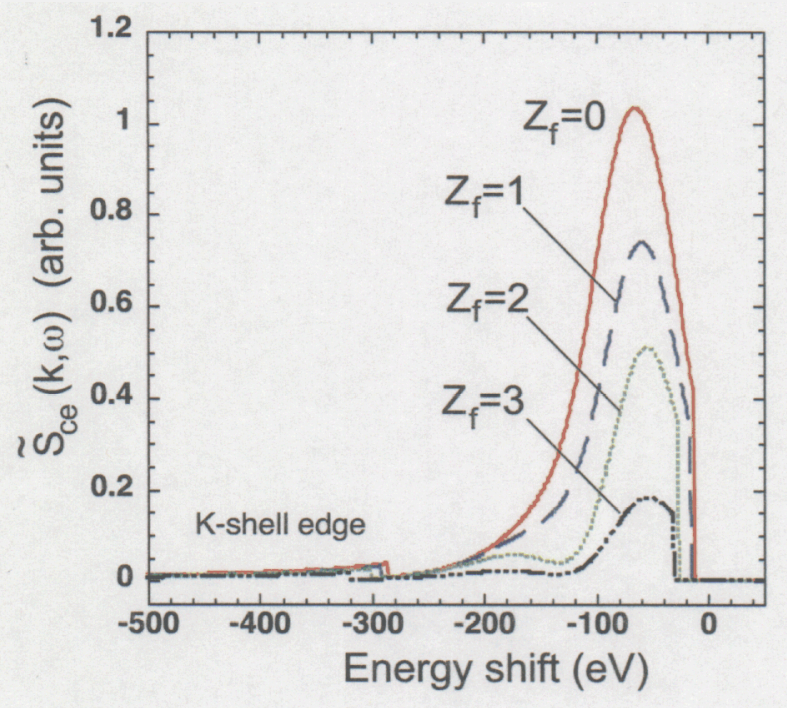

FIG. 1: (color) Calculated bound-free dynamic structures, $\tilde{S}_{c e}(k, \omega)$, for a carbon plasma with density $0.72 \mathrm{~g} / \mathrm{cc}$ at $130^{\circ}$ scattering angle. The probe energy is $E_{0}=4.75 \mathrm{keV}$. The ionization energy is corrected for continuum lowering.

a finite-temperature Thomas-Fermi model which reproduces both the classical Debye screening for low density plasmas and the ion-sphere correlation length for high density coupled systems. In their model, the lowering of the ionization potential is given by:

$$
\Delta E_{B}=\frac{Z_{f} e^{2}}{\left(4 \pi \epsilon_{0}\right) \lambda_{s}}
$$

with,

$$
\lambda_{s}=\frac{2}{3} \frac{Z_{f}^{1 / 3} d\left(\frac{z_{f}^{1 / 3} d}{\lambda_{D}}\right)^{2}}{\left[\left(\frac{z_{f}^{1 / 3} d}{\lambda_{D}}\right)^{3}+1\right]^{2 / 3}-1}
$$

The mean radius per electron is $d=\left(3 / 4 \pi n_{e}\right)^{1 / 3}$, and $\lambda_{D}$ is the Debye length. Since our experimental conditions are also relevant in the investigation of degenerate electron systems, the Debye length should be calculated as $\lambda_{D}=\sqrt{\epsilon_{0} k_{B} T_{c f} / n_{e}\left(Z_{f}+1\right) e^{2}}[1]$, where the effective temperature $T_{c f}=\left(T_{e}^{2}+T_{q}^{2}\right)^{1 / 2}$, with $T_{q}=$ $T_{F} /\left(1.3251-0.1779 \sqrt{r_{s}}\right), T_{F}$ the Fermi temperature, and $r_{s}=d / a_{B}$. This corrected temperature is chosen such that the temperature of an electron liquid obeying classical statistics exactly gives the same correlation energy of a degenerate quantum fluid at $T_{e}=0$ obtained from quantum Monte Carlo calculations [30]. This approach was shown to reproduce finite-temperature static response of an electron fluid, valid for arbitrary degeneracy [30]. For typical experimental conditions, $\Delta E_{B}$ accounts for $\sim 30-50 \%$ of the ionization energy, thus continuum lowering strongly shifts the L-shell edges of the bound-free dynamic structure. 


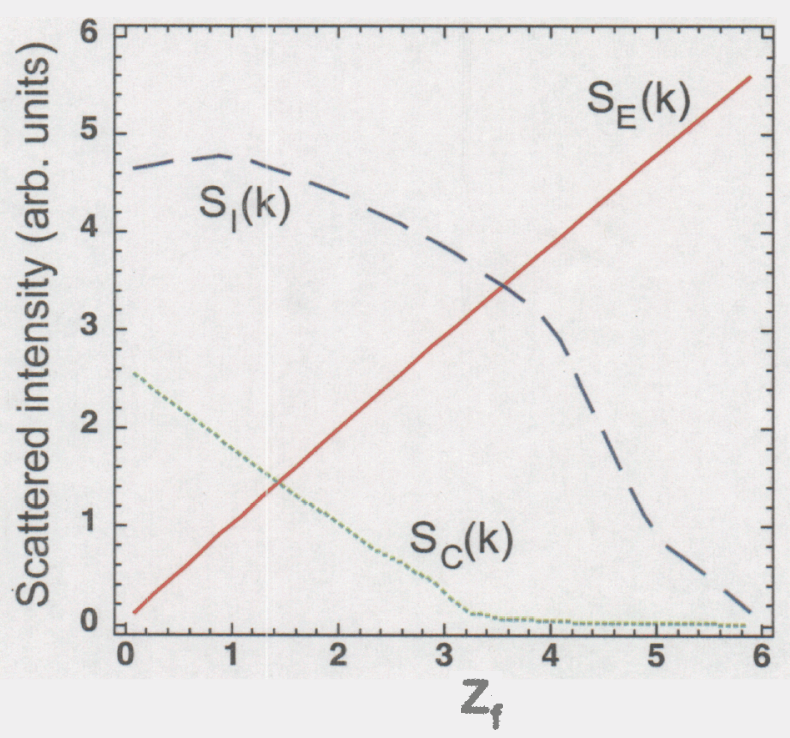

FIG. 2: (color) Static structures obtained for a carbon plasma with density $0.72 \mathrm{~g} / \mathrm{cc}$ and $T_{e}=20 \mathrm{eV}$. The probe radiation is $E_{0}=4.75 \mathrm{keV}$ and the scattering angle is $130^{\circ}$.

It is interesting to compare the total scattered power by bound and free electrons in the elastic and inelastic terms. By integrating in frequency Eq. (3) we obtain the total static structure

$$
S(k)=S_{I}(k)+S_{E}(k)+S_{C}(k),
$$

where,

$$
\begin{gathered}
S_{I}(k)=\left|f_{I}(k)+q(k)\right|^{2} S_{i i}(k), \\
S_{E}(k)=Z_{f} S_{e e}^{0}(k),
\end{gathered}
$$

and,

$$
S_{C}(k)=Z_{c} \int_{e E_{B} / \hbar}^{e E_{0} / \hbar} \tilde{S}_{c e}(k, \omega) d \omega
$$

The static structures $S_{i i}(k)$ and $S_{e e}^{0}(k)$ can be easily obtained through the prescription described in Ref. [1]. The total elastic component of the scattered x-ray radiation is $S_{I}(k)$ and it includes contribution from both free and bound electrons. Inelastic scattering by free electrons and bound electrons is given by the terms $S_{E}(k)$ and $S_{C}(k)$, respectively. In Figure 2 we have plotted as a function of the charge state the various static structure for a carbon plasma, $0.72 \mathrm{~g} / \mathrm{cc}, T_{e}=20 \mathrm{eV}$, probed with x-rays of energy $E_{0}=4.75 \mathrm{keV}$ at $130^{\circ}$ scattering angle. We see that for $Z_{f} \lesssim 3$, the amount of the scattered radiation by bound-free transitions is quite large and it must be considered for a correct evaluation of the experimental profiles, if accurate measurements of the electron density, electron temperature and charge state need to be performed.

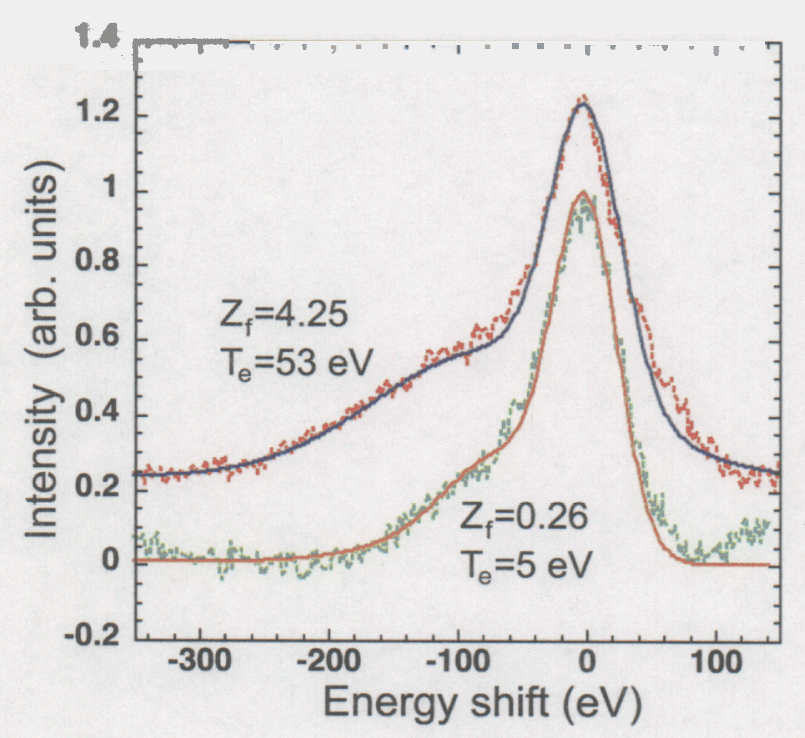

FIG. 3: (color) Experimental x-ray scattering data from a heated carbon foam $(0.72 \mathrm{~g} / \mathrm{cc})$ and a cold carbon foam. The probe radiation is the $\mathrm{Ti} \mathrm{He}-\alpha$ line at $4.75 \mathrm{keV}$, and the scattered $x$-rays are collected at $\sim 130^{\circ} \pm 5^{\circ}$ scattering angle. Best fit parameters and corresponding spectra are also plotted in the figure. For the high temperature foam, $\alpha=0.17$, $T_{F}=10.4 \mathrm{eV}$, and $\Gamma=0.2$; while for the cold foam $\alpha=0.13$, $T_{F}=1.6 \mathrm{eV}$, and $\Gamma=0.9$.

\section{EXPERIMENTAL RESULTS}

We apply the technique discussed in the previous section to a dense carbon plasma. We used the $30-\mathrm{kJ}$ Omega laser facility [31] to produce a homogeneous and isochorically heated carbon plasma at solid density, and then probed the plasma interior with the $\mathrm{Ti} \mathrm{He}-\alpha$ x-ray line at $4.75 \mathrm{keV}$ from a secondary laser produced plasma. The details of the experimental technique have been extensively discussed by Glenzer et al. [2, 3] and we will refer the reader to those papers for additional information. Prior to laser heating, the carbon is in an amorphous (foam) state with density $0.72 \mathrm{~g} / \mathrm{cc}$. Since during the heating time the plasma is not expanding (see Ref. [3]), the initial carbon density sets the ion density to $n_{i}=3.6 \times 10^{22} \mathrm{~cm}^{-3}$. The electron density is then determined by the ionization state of the system. By changing the number of driver beams on the foam, we can vary the degree of carbon heating and consequently its ionization state.

In Figure 3 we have plotted experimental profiles obtained for two different cases: a strongly heated foam and a cold one. The scattered radiation has been collected at $\sim 130^{\circ} \pm 5^{\circ}$ scattering angle with a high efficiency graphite Bragg crystal operated in mosaic focusing mode $[2,3]$. This geometry corresponds to a scattering parameter $\alpha=1 / k \lambda_{D e}<1$, where $\lambda_{D e}=\sqrt{\epsilon_{0} k_{B} T_{c f} / n_{e} e^{2}}$. Thus the scattering is noncollective and the spectra of the free electrons directly show the distribution function [1]. 

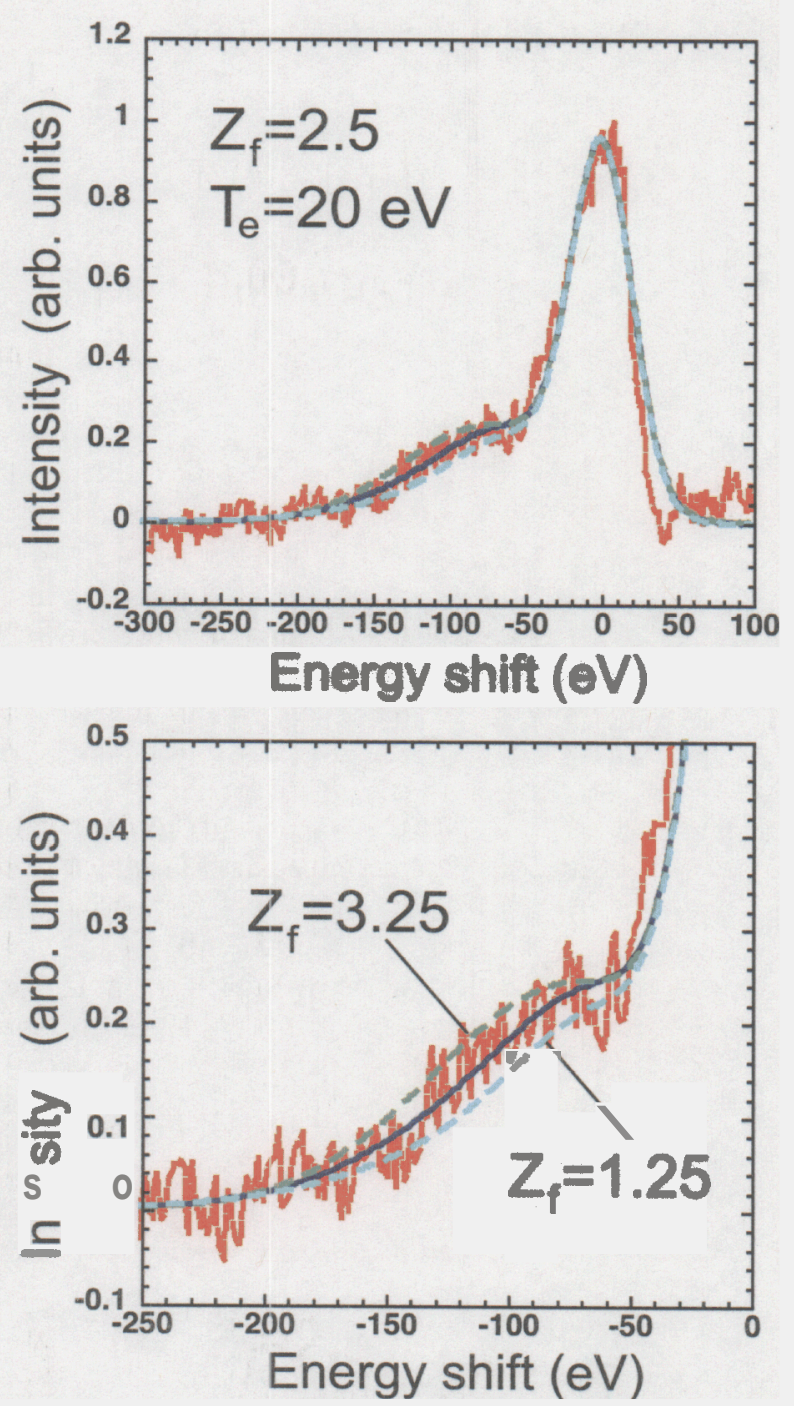

FIG. 4: (color) Experimental x-ray scattering data from a heated carbon foam $(0.72 \mathrm{~g} / \mathrm{cc})$ and a cold carbon foam. The probe radiation is the $\mathrm{Ti} \mathrm{He}-\alpha$ line at $4.75 \mathrm{keV}$, and the scattered x-rays are collected at $\sim 130^{\circ} \pm 5^{\circ}$ scattering angle. Best fit parameters and corresponding spectra are also plotted in the top panel. The comparison between different ionization state theoretical lineshapes with the data is zoomed in the bottom panel. For these experimental conditions $\alpha=0.21$, $T_{F}=7.3 \mathrm{eV}$, and $\Gamma=0.5$.

From the figure we notice an increased red wing for the higher temperature foam, indicating that a larger number of electrons have been downshifted in energy by the Compton effect. Since these are essentially free electrons, an increased red wing in the spectrum is thus a signature of a higher ionization state. By combining the theory outlined in the previous section for the core electrons with the approach described by Gregori et al. [1] based on the RPA for the free electron density response, we can fit the experimental data to obtain $T_{e}$ and $Z_{f}$. The electron density is then simply given as $n_{e}=Z_{f} n_{i}$, as heating is

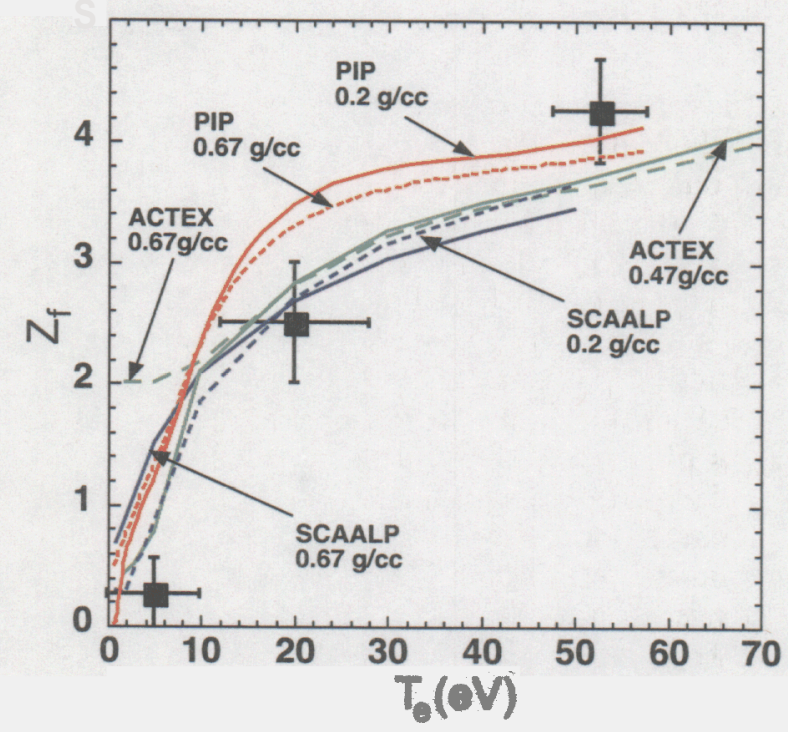

FIG. 5: (color) Temperature-ionization diagram along with the results of the $x$-ray scattering measurements and various EOS models (see text).

isochoric and the plasma does not expand at the probing time. The high temperature foam gives $Z_{f}=4.25$ and $T_{e}=53 \mathrm{eV}$, while for the cold foam $Z_{f}=0.26$ and $T_{e}=5 \mathrm{eV}$. As discussed by Glenzer et al. [2], the error in the temperature measurement for the high temperature foam is $\$ 20 \%$. For the cold foam, instead, the electron plasma is fully degenerate $\left(T_{e} \sim T_{F}\right)$ and the width of the Compton feature is only weakly sensitive on the electron temperature. Moreover, the Compton profile mainly results from bound-free transitions which directly reflects the heavy ion temperature. Under these conditions, the fitted temperature is understood only as an upper limit of the actual electron temperature of the degenerate electron fluid. As a final remark, we notice that for our experimental conditions, the electron-electron coupling constant $\Gamma=e^{2} / 4 \pi \epsilon_{0} k_{B} T_{c f} d \lesssim 1$, thus local field corrections to the RPA are not important [8].

In Figure 4 it is plotted the $x$-ray scattering spectrum from a moderately heated foam. The best fit values for this case are $Z_{f}=2.5$ and $T_{e}=20 \mathrm{eV}$, and the corresponding theoretical form factor, convoluted with the appropriate instrument function, is also reported in the Figure. In order to test the sensitivity in the ionization state measurement, we have plotted theoretical spectra for two different values of $Z_{f}$, all the other parameters being the same. The Figure shows that a typical error in the ionization state value is $\sim \pm 0.5$.

Figure 5 shows the $T_{e}-Z_{f}$ phase diagram along with experimental data and various EOS models for carbon. These are the activity expansion method (ACTEX) $[32,33]$, the partially ionized plasma (PIP) model $[34,35]$ and SCAALP, a density functional plasma model [36]. In the ACTEX theory, all possible interactions between 
plasma constituents are calculated including the screening of the bound states. For large densities, the classical Debye-Hückel (Yukawa) potential is replaced by a screened potential which has a cut-off for distances that approach the thermal de Broglie wavelength, in order to mimic quantum mechanical effects (i.e., exchange and symmetry). This approach allows the calculation of delocalized electrons, i.e., the number of electrons that are no longer bound to a single ion. These electrons are free or weakly bound like the conduction electrons in a metal. For our conditions, these electrons give rise to the Compton downshifted electron feature of the $\mathrm{x}$-ray scattering spectrum. The PIP model is based on the self-consistent solution of Saha-like equations for each ionization stage together with the calculation of appropriate chemical potentials for electrons and ions. This also allows the inclusion of high density effects by using corrected chemical potentials for the continuum lowering. SCAALP is based on the density functional theory for plasmas, where electronic structure and ionic distribution are determined self-consistently. The plasma is considered as an effective classical system of virtual neutral particles (neutral pseudo-atom, NPA) interacting via an interatomic effective potential $V_{\text {eff }}(r)$. Electrons of the NPA satisfy a Schrodinger equation with an effective central symmetric potential $\phi(r)$. Both $V_{\text {eff }}$ and $\phi$ are determined by the electronic structure and the ionic distribution of the plasma. Polarization and correlation effect of the continuum electrons are taken into account, as well as a part of the exchange interaction within $V_{\text {eff }}$.

Results from these models, assuming different values for the carbon density, are plotted in Figure 5. The comparison with the experimental data shows good agreement with SCAALP at all densities, even if some differences still remain especially for the high temperature case. The PIP model also gives reasonably good agreement with the data at all densities, but it seems to over-predict the ionization state in the mid-temperature regime. In this regime the plasma undergoes a transition from a degenerate fluid to a classical one, thus a full quantum mechanical treatment beyond the Saha description may be required. ACTEX shows a similar trend to SCAALP for the low density simulation, but, in the higher density case, predicts a low temperature foam which still have $\sim 2$ electrons in the conduction band.
This transition to a metallic state for carbon at high density are not reproduced by the other models and our experimental data at low $T_{e}$ also shows an insulating behavior for carbon at high density.

From this discussion, we see that currently available EOS models for carbon exhibit different behavior in the temperature range $0-50 \mathrm{eV}$, which span the range from fully degenerate to classical plasmas. X-ray scattering thus provides an accurate experimental tool for validation and improvement of EOS codes, as clearly shown in Figure 5.

\section{CONCLUSIONS}

In this paper we have given approximate expressions to calculate the $\mathrm{x}$-ray scattering form factor from weakly bound electrons which are accurate for moderate to low- $Z$ materials. The approach that we have followed is based on the IA corrected for the asymmetry induced by the electron binding. Together with the RPA for the free electron dynamic structure, we were able to obtain a full description of the $x$-ray scattering form factor for a carbon plasma. We have compared our model with experimental data from the Omega laser facility in order to extract accurate values for electron temperature and ionization state. This has allowed the comparison between various ionization balance models for carbon with our data, thus enabling a direct validation of EOS theories for a carbon plasma in a regime which cover the transition between a degenerate to classical fluid. Our result are of interest for ICF research as well as planetary science since they indicate that matter under extreme conditions, as the one found in fuel pellet during compression or in the interior of planets, can now be investigated with good accuracy.

\section{Acknowledgments}

This work was performed under the auspices of the U.S. Department of Energy by the University of California Lawrence Livermore National Laboratory under Contract No. W-7405-ENG-48. We also acknowledge support from Laboratory Directed Research and Development grant No. 02-ERD-13.
[1] G. Gregori, S. H. Glenzer, W. Rozmus, R. W. Lee, and O. L. Landen, Phys. Rev. E 67, 026412 (2003).

[2] S. H. Glenzer, G. Gregori, R. W. Lee, F. J. Rogers, S. W. Pollaine, and O. L. Landen, Phys. Rev. Lett. 90, 175002 (2003).

[3] S. H. Glenzer, G. Gregori, F. J. Rogers, D. H. Froula, S. W. Pollaine, R. S. Wallace, and O. L. Landen, Phys. Plasmas p. in press (2003).

[4] J. D. Lindl, Inertial Confinement Fusion (SpringerVerlag, New York, 1998).
[5] D. Pines and D. Bohm, Phys. Rev. 85, 338 (1952).

[6] D. Pines and P. Nozieres, The Theory of Quantum Fluids (Addison-Wesley, Redwood, CA, 1990).

[7] S. Ichimaru, Rev. Mod. Phys. 54, 1017 (1982).

[8] G. Gregori, S. H. Glenzer, and O. L. Landen, J. Phys. A: Math Gen. 36, in press (2003).

[9] J. Chihara, J. Phys. F: Met. Phys. 17, 295 (1987).

[10] J. Chihara, J. Phys.: Condens. Matter 12, 231 (2000).

[11] D. Riley, N. C. Woolsey, D. McSherry, I. Weaver, A. Djaoui, and E. Nardi, Phys. Rev. Lett. 84, 1704 
(2000).

[12] S. Ichimaru, Basic Principles of Plasma Physics (Addison, Reading, MA, 1973).

[13] E. E. Salpeter, Phys. Rev. 120, 1528 (1960).

[14] D. E. Evans and J. Katzenstein, Rep. Prog. Phys. 32, 207 (1969).

[15] P. Eisenberger and P. M. Platzman, Phys. Rev. A 2, 415 (1970).

[16] R. Currat, P. D. DeCicco, and R. Kaplow, Phys. Rev. B 3, 243 (1971).

[17] J. W. M. DuMond, Phys. Rev. 29, 643 (1929).

[18] B. J. Bloch and L. B. Mendelsohn, Phys. Rev. A 12, 1197 (1975).

[19] L. Pauling and J. Sherman, Zeit. f. Krist. 1, 81 (1932).

[20] P. M. Bergstrom and R. H. Pratt, Radiat. Phys. Chem. 50, 3 (1997).

[21] A. Issolah, B. Levy, A. Beswick, and G. Loupias, Phys. Rev. A 38, 4509 (1988).

[22] F. Bell, J. Chem. Phys. 85, 303 (1986).

[23] B. J. Bloch and L. B. Mendelsohn, Phys. Rev. A 9, 129 (1974).
[24] F. Gasser and C. Tavard, Phys. Rev. A 27, 117 (1983).

[25] P. Holm and R. Ribberfors, Phys. Rev. A 40, 6251 (1989).

[26] Y. F. Chen, C. M. Kwei, and C. J. Tung, Phys. Rev. A 47, 4502 (1993).

[27] T. Paakkari and P. Suortti, Phys. Rev. B 9, 1756 (1974).

[28] R. W. James, The Optical principles of the Diffraction of $X$-rays (Ox Bow Press, Woodbridge, CT, 1962).

[29] J. C. Stewart and K. D. Pyatt, ApJ 1203, 144 (1966).

[30] M. W. C. Dharma-Wardana and F. Perrot, Phys. Rev. Lett. 84, 959 (2000).

[31] J. M. Soures et al., Fusion Technol. 30, 492 (1996).

[32] F. J. Rogers and D. A. Young, Phys. Rev. E 56, 5876 (1997).

[33] F. J. Rogers, Phys. Plasmas 7, 51 (2000).

[34] S. Kuhlbrodt and R. Redmer, Phys. Rev. E 62, 7191 (2000).

[35] R. Redmer, Phys. Rev. E 59, 1073 (1999).

[36] P. Renaudin, C. Blancard, G. Faussurier, and P. Noiret, Phys. Rev. Lett. 88, 215001 (2002). 